\title{
Desarrollo de procesos reflexivos desde la percepción de estudiantes de enfermería
}

\author{
Development of Reflexive Processes from the \\ Perception of Nursing Students
}

\author{
Jessica Olate \& Silvana Castillo \\ Universidad de Chile, Santiago, Chile
}

\begin{abstract}
Resumen: Este estudio se realizó al finalizar una asignatura impartida en segundo año de enfermería, cuyo propósito fue contribuir a la formación integral del estudiante, a través del autoconocimiento y desarrollo de habilidades sociales para favorecer el establecimiento de la relación terapéutica. Para ello, se utilizaron estrategias que intencionan el desarrollo de la reflexión, y se indagó en la percepción de los estudiantes sobre sus procesos reflexivos. El objetivo era explorar cómo los estudiantes de enfermería perciben el desarrollo de sus procesos reflexivos después de participar en el curso. Es una investigación naturalista, que utilizó como método cualitativo el estudio de caso único e intrínseco; se realizaron entrevistas semiestructuradas a los estudiantes, previa firma de consentimiento informado. Se desarrolló un análisis de datos inductivo, mediante el método comparativo constante. Como resultados emergieron tres núcleos temáticos relacionados: desarrollo del proceso reflexivo, experiencias de trabajo grupal, y desarrollo de aprendizaje significativo. Se concluye que el proceso reflexivo de los estudiantes corresponde a un fenómeno recursivo que se va profundizado y madurando en el tiempo, siendo percibido como una habilidad que puede ser intencionada en la formación y resulta útil a nivel personal, académico y profesional futuro.
\end{abstract}

Palabras clave: proceso reflexivo, reflexión, formación enfermería.

\begin{abstract}
This research was performed at the end of a subject taught in the second year of Nursing, whose purpose was to contribute to the integral formation of the student, through self-knowledge and development of social skills to favor the establishment of therapeutic relationships. Strategies were used to intend the development of the reflection, and it was pertinent to investigate the perception of the students on their own reflective processes. The aim was to explore how nursing students perceive the development of their reflective processes. It was a naturalistic research, which used a qualitative method to study a single and intrinsic case; semi-structured interviews were carried out, after signing an informed consent by students. An inductive data analysis was performed using the constant comparison method. As results three related thematic groups emerged: development of the reflective process, team work experiences and development of meaningful learning. It was concluded that reflective processes of nursing students correspond to a recursive phenomenon that become deep and mature over time. This process is perceived as the development of a skill, which is identified as useful not only in a personal level, but also as an academic tool and as future professionals.
\end{abstract}

Keywords: reflective process, reflection, nursing training.

Contacto: J. Olate. Departamento de Enfermería, Universidad de Chile, Avenida Independencia 1027, Independencia, Santiago, Chile. Código postal: 8930610. Correo electrónico: jolate@ med.uchile.cl

Cómo citar: Olate, J. \& Castillo, S. (2016). Desarrollo de procesos reflexivos desde la percepción de estudiantes de enfermería. Revista de Psicología, 25(2), 1-18.

http://dx.doi.org/10.5354/0719-0581.2016.44775 


\section{Introducción}

La formación actual de profesionales de enfermería necesita una transformación radical, dado los requerimientos para un desempeño seguro y preciso, en un entorno variable y complejo, donde los conocimientos y la innovación avanzan a un ritmo acelerado. Estos profesionales deben iniciar su práctica profesional dispuestos a seguir aprendiendo, a ampliar sus áreas de conocimientos y, por otra parte, a trabajar bajo circunstancias y entornos complicados, debiendo desplegar un conjunto complejo de habilidades y conocimientos, junto a un profundo compromiso con cada uno de sus pacientes (Benner, Surphen, Leonard, \& Day, 2010).

Un foco de discusión que ha surgido en la formación de profesionales de enfermería tiene relación con la reflexión como elemento esencial de la práctica profesional, ya que una estructura reflexiva consistente durante los procesos de formación puede facilitar el aprendizaje y el uso de la reflexión de los estudiantes de enfermería (Asselin, 2011). Esta formación de enfermeras/os se debe desarrollar en conexión con el progreso de la investigación y el conocimiento pedagógico, como respuesta a las transformaciones complejas de la práctica profesional (Medina Moya, 2002).

Por su parte, la Guía de buenas prácticas en enfermería (Registered Nurses' Association of Ontario [RNAO], 2006) plantea que los/las profesionales de enfermería, para establecer una relación terapéutica y brindar cuidados humanizados, deben realizar una práctica clínica reflexiva, que considera el desarrollo de capacidades como la autoconciencia, autoconocimiento y comprensión del alcance y los límites de la labor profesional, las que inicialmente están asociadas al desarrollo de la reflexión, que debe intencionarse en la etapa de formación profesional.

La Escuela de Enfermería de la Universidad de Chile lleva varios años incorporando progresivamente estrategias de enseñanza reflexiva en sus cursos de pregrado. Uno de los pioneros es el curso de Interacción humana y comunicación, que tiene como propósito contribuir al desarrollo personal del estudiante de enfermería, a través del autoconocimiento y mejora de habilidades sociales, con el fin de favorecer el establecimiento de una relación terapéutica óptima y constructiva. En esta experiencia, se intenciona el desarrollo de la reflexión en los estudiantes de enfermería, a partir de lo cual surge la interrogante de investigación: ¿cómo perciben los estudiantes de enfermería el desarrollo de sus procesos reflexivos después de participar en la asignatura de Interacción humana y comunicación?

El propósito de este estudio es plantear temas de discusión relacionados con la formación de profesionales de enfermería reflexivos y proponer mejoras a las estrategias formativas vinculadas, basándose en los resultados de la investigación.

\section{Objetivos del estudio}

\section{General}

Explorar cómo los estudiantes de enfermería perciben el desarrollo de sus procesos reflexivos después de participar en la asignatura de Interacción humana y comunicación.

\section{Específicos}

1) Describir desde la percepción de los estudiantes, las instancias en que lograron desarrollar procesos de reflexión, 
en el contexto de la asignatura de Interacción humana y comunicación.

2) Indagar en la valoración que los estudiantes le atribuyen al proceso reflexivo como contribución a su formación personal y profesional.

3) Comprender la percepción de los estudiantes, respecto al uso del diario reflexivo como estrategia metodológica.

4) Identificar los aspectos de la asignatura que los estudiantes perciben como facilitadores para el desarrollo de sus procesos reflexivos.

5) Identificar los aspectos de la asignatura que los estudiantes perciben como obstaculizadores para el desarrollo de sus procesos reflexivos.

\section{Desarrollos teóricos}

Los problemas que los profesionales deben enfrentar en la realidad no siempre son estructuras bien organizadas; muchas veces se trata de problemas más complejos de resolver y que se presentan como situaciones poco definidas (Schön, 1992). El profesional debe construir el problema, definirlo para que encaje con las teorías o técnicas que mejor conoce y recién entonces poder afrontarlo. Desde este punto de vista, para que el profesional en la práctica convierta una situación problemática e incierta en un problema bien definido, no utiliza en primera instancia la solución técnica o saber teórico abstracto, sino que esta se realiza en forma posterior a la construcción del problema, la que se basa en el conocimiento práctico. Bajo esa misma lógica Schön utiliza la denominación de práctico reflexivo, cuyas acciones están basadas en un conocimiento práctico y tácito que se activa frente a situaciones ambiguas; dicho proceso puede distinguirse por tres componentes: conocimiento en la acción, reflexión en la acción y reflexión sobre la reflexión en la acción (Schön, 1992).
Al aplicar la formación del práctico reflexivo en los profesionales de enfermería, sería atingente promover un aprendizaje integrador, que considere la reflexión sobre la práctica para ayudar al estudiante a desarrollar una práctica de mejoramiento personal (Benner et al., 2010). El aprendizaje experiencial se genera desde una experiencia que el/la estudiante observa y sobre la cual reflexiona, realizando una abstracción e integrando estas reflexiones en su mente, para utilizarlas como guías en acciones posteriores (Medina Moya, 2002).

Las investigaciones de Patricia Benner (1984) demuestran la existencia de un conocimiento implícito que se activa con la acción de la enfermera/o y que permite el reconocimiento instantáneo de situaciones problemáticas y respuestas intuitivas que son características de una práctica profesional experta. Las/os enfermeras/os expertas/os pueden reconocer rápidamente situaciones irregulares en las personas que cuidan, sin embargo, no pueden presentar una descripción precisa del método o proceso racional que han seguido para alcanzar esa conclusión (Medina Moya, 2008).

Al profundizar sobre el actuar de las enfermeras expertas, ellas se distinguen por características de liderazgo, habilidades de supervisión, gestión del cambio, habilidades comunicacionales y relacionales elevadas, capacidad de actuar reflexivamente, planificar, organizar, evaluar y tener más destreza que las enfermeras novicias. También poseen mayor capacidad de adaptación, seguridad, visión global, competencia en el manejo de conflictos, manejo del estrés y de articulación entre teoría y práctica; junto con ser capaces de crear conocimiento mediante la investigación, y responder a situaciones complejas con capacidad de decisión. Las enfermeras expertas se destacan por la capacidad de 
anticipación, perspicacia, observación detallada, acción rápida y la competencia en la fijación de prioridades, visión de contexto y tendencia a la especialización (Pina Queirós, 2015).

Los antecedentes antes expuestos dejan al descubierto la importancia de la formación de pregrado para el desarrollo futuro desde un profesional de enfermería novicio a experto. Es necesario que los docentes de enfermería sean reflexivos y capaces de fomentar la reflexión de los estudiantes, para que sus procesos de enseñanza-aprendizaje sean significativos y potencien su desarrollo como profesionales reflexivos. El formador reflexivo no prepara a estudiantes reflexivos con solo mostrar una postura concordante, sino que se requiere una intención y estrategias centradas en el entrenamiento para la reflexión y el análisis de diferentes ámbitos del conocimiento y las competencias (Perrenoud, 2004).

La reflexión facilita la creación de sentido, en la medida que permite renunciar a las evidencias y a los problemas definitivamente resueltos; fomentando una actitud de búsqueda y cuestionamientos, y reconociendo la complejidad e incertidumbres de las interacciones y sus consecuencias (Perrenaud, 2004). Sin embargo, para desarrollar una postura reflexiva es necesario formar un habitus, es decir, adquirir esquemas reflexivos que a lo largo de la vida puedan desarrollarse y ajustarse de acuerdo a las situaciones que experimente el profesional. La gran importancia que adquiere el habitus radica, principalmente, en que gran parte de las acciones profesionales se realizan de manera inmediata o improvisando intuitivamente, no existiendo un tiempo para detenerse a pensar en los saberes disponibles para la acción requerida, "formar buenos principiantes es, precisamente, formar de entrada a gente capaz de evolucionar, de aprender con la experiencia, que sean capaces de reflexionar sobre lo que querían hacer, sobre lo que realmente han hecho y sobre el resultado de ello" (Perrenoud, 2004, p. 17).

Entonces, se puede decir que existe una relación entre la formación de profesionales de enfermería reflexivos y un perfil docente que debe fomentar en los estudiantes aprendizajes significativos, habilidades de pensamiento superior $\mathrm{y}$ capacidades para aprender a aprender, a través de la revisión del ejercicio profesional y la habilidad de pensamiento reflexivo. Este perfil docente también debe relacionarse con programas de formación que estimulen el desarrollo de procesos de reflexión y que se enfoquen en la enseñanza reflexiva, fomentando en los estudiantes habilidades metacognitivas que les permitan interrogar, analizar, conocer, evaluar y modificar su propia práctica desde un punto de vista profesional, moral y social (Medina Moya, Jarauta Borrasca, \& Imbernon Muñoz, 2010).

Relacionado con lo anterior, Dahl y Eriksen (2016) aplican una estrategia para mejorar los procesos de reflexión de los estudiantes y plantean como aspectos relevantes el detenerse y verbalizar las experiencias, estimular y apoyar los procesos de reflexión, y el desarrollo de una actitud consciente por parte de los estudiantes al darse cuenta de ellos mismos y de su propia ejecución. Los docentes y grupos de reflexión se consideran cruciales al momento de aprender a ver la complejidad de una determinada situación y cumplir un rol de ayuda en la conexión entre la situación y los conocimientos teóricos. Este estudio concluye que mejorar el desarrollo profesional de los estudiantes requiere prestar atención a las habilidades de facilitación docente. 
Sin embargo, Domingo (2013) plantea que existe escasa evidencia sistematizada de la formación relacionada con la práctica reflexiva que permita implementarla de manera formal en el currículum universitario. Por esto desarrolló una investigación en el contexto de la formación de profesores, en que, a partir de un diagnóstico, se elabora, implementa y evalúa un modelo formativo basado en la práctica reflexiva, denominado Método R. Dentro de las conclusiones derivadas del estudio, se evidencia que la implementación de este método favorece en el estudiante el desarrollo de la competencia reflexiva, experimentado como una espiral continua de acción-reflexión-acción, que permite la toma de decisiones en contextos complejos y la capacidad de replantear su propio desempeño.

Por esto, se hace necesario explicitar la importancia de intencionar, desde los programas curriculares y las metodologías docentes, el desarrollo de procesos reflexivos en los estudiantes para el fomento de aprendizajes significativos y habilidades de metacognición, elementos fundamentales en profesionales reflexivos. Aprender significativamente implica construir un significado propio y personal para un objeto de conocimiento que existe objetivamente. En dicho proceso, no solamente se modifica lo que ya se posee, sino que además hay un proceso particular de interpretación de lo nuevo, de manera que se pueda integrar y hacerlo propio (Coll et al., 2005).

Existen diferencias sustanciales relacionadas con las capacidades reflexivas de los estudiantes y que pueden asociarse al proceso cíclico de aprendizaje, que está compuesto por las fases activa, reflexiva, teórica y pragmática, identificando cuatro estilos de aprendizajes del mismo nombre que sugieren que el docente pueda planificar actividades que beneficien al mayor número posible de estudiantes pasando por las distintas fases y repitiendo el ciclo, enfocado en una mejora continua del proceso de enseñanza-aprendizaje, pero, a la vez, tomando en cuenta las características individuales de los estudiantes (Lago, Colvin, \& Cacheiro, 2008).

Llevar lo anterior a la práctica se puede realizar a través del uso del cuestionario de Honey-Alonso de Estilos de Aprendizaje (CHAEA), que permite tanto a los estudiantes como al docente individualizar los estilos de aprendizaje e identificar aquellos más desarrollados y los que requieren potenciarse, con el fin de diseñar herramientas pedagógicas que contemplen estos aspectos para favorecer el rendimiento académico (González, Sáez, \& Ramírez, 2016). Desde el punto de vista del desarrollo del estilo reflexivo, es necesario comprender la reflexión como un proceso dinámico y progresivo, que, según Medina Moya et al. (2010), posee tres fases de desarrollo:

1) Conflictos entre los conocimientos y los sentimientos aplicados a una situación, y la situación misma: frente a una situación, se toma conciencia de que el conocimiento que se está aplicando no es suficiente para responder.

2) Cuestionamiento de los conocimientos y los sentimientos: contraste de los conocimientos disponibles mediante la elaboración de comprensiones específicas durante el propio proceso de actuación.

3) Modificación de la comprensión de la situación: construcción de una nueva visión o perspectiva de la situación en términos de aprendizaje.

Existen diversas propuestas para intencionar el desarrollo del proceso reflexivo en la formación de profesionales y para efectos de este estudio se contemplan los 
planteamientos de Brockbank \& Mcgill (2002), quienes sugieren reforzar la calidad del proceso educativo para promover un aprendizaje adecuado en la enseñanza superior. Dicha propuesta se basa en los planteamientos de Schön (1992) respecto a la formación de profesionales reflexivos, explicando el concepto de reflexión y práctica reflexiva, realizando aportes desde el punto de vista de la aplicación y además, posicionando el concepto de diálogo reflexivo intencionado para facilitar el aprendizaje y la práctica reflexiva del estudiante (Brockbank \& Mcgill, 2002).

\section{Reflexión}

Proceso por el que se tiene en cuenta la experiencia y la creación de significado y la conceptualización a partir de la experiencia y la capacidad de mirar las cosas como potencialmente distintas de como aparecen, incluyendo en este último aspecto la idea de la reflexión crítica. Cuando se considera la experiencia, se incluye el pensamiento, el sentimiento y la acción; cuando la reflexión es intencionada, con el fin de promover el aprendizaje, el aprendiz incluye esa acción en el diálogo (Brockbank \& Mcgill, 2002). En relación con la reflexión como elemento de la práctica reflexiva y dentro de este diálogo reflexivo, la integración de mente y cuerpo (afecto y acción) significa que aportamos al acto de la reflexión nuestra experiencia cognitiva y afectiva (Brockbank \& Mcgill, 2002).

\section{Diálogo reflexivo intencionado}

Permite el aprendizaje críticamente reflexivo, compromete a la persona hasta el extremo de sus conocimientos, su sentido del yo y del mundo, tal como lo experimentamos y cuya intención consiste en proporcionar un contexto para el aprendizaje reflexivo y apoyarlo (Brockbank \& Mcgill, 2002).

\section{Práctica reflexiva}

Saber qué. Lo que adquiere el estudiante profesional de su estudio científico en la universidad, es decir, saber de libro o saber acerca de (Brockbank \& Mcgill, 2002).

Saber en la acción y saber al uso. Procede de la práctica profesional. Schön (1992) lo llama "conocimiento en la acción" y produce resultados en la medida en que la situación está dentro de los límites de lo que hayamos aprendido a considerar normal (Brockbank \& Mcgill, 2002; Schön, 1992).

Reflexión en la acción. Se produce cuando nos encontramos en medio de una acción y, al hacerla y mostrarnos reflexivos, decidimos. Para Schön (1992), lo que distingue la reflexión en la acción de otras formas de reflexión es:

Su significación inmediata para la acción, el replanteamiento de alguna parte de nuestro saber en la acción lleva a un experimento sobre la marcha y a nuevos pensamientos que influyen en lo que hagamos, en la situación en la que nos encontremos y quizá también en otras que nos parezcan similares a ella (p. 29).

Esto significa reflexionar mientras está produciéndose la acción (Brockbank \& Mcgill, 2002).

Reflexión sobre la acción. Reflexión de nivel superior, en un metanivel en que el alumno es capaz, en primer lugar, de describir o nombrar lo ocurrido, y después de reflexionar sobre ello, es decir de trabajar con el material que tiene ante sí, la reflexión sobre la acción se convierte en un saber al uso potencial (Brockbank \& Mcgill, 2002). 
Reflexión sobre la reflexión sobre la acción. El grupo reflexiona sobre la reflexión sobre la acción en el diálogo reflexivo. El individuo puede aprender gracias a la interacción en el grupo, es decir, es aprendizaje sobre el aprendizaje (Brockbank \& Mcgill, 2002).

\section{Contexto del estudio}

El estudio se desarrolló al finalizar la asignatura de Interacción humana y comunicación el año 2013. Esta fue impartida en el tercer semestre de la carrera de enfermería, y su propósito era contribuir al desarrollo personal del estudiante, a través del autoconocimiento y desarrollo de habilidades sociales, para su formación integral en beneficio de una relación terapéutica óptima.

Los objetivos de este curso son: desarrollar habilidades para establecer relaciones interpersonales satisfactorias durante la formación académica, como base para el desempeño profesional futuro $\mathrm{y}$; reflexionar en torno a la vivencia individual y grupal, durante el desarrollo de las sesiones grupales.

Para dar cumplimiento a los objetivos planteados, el curso fue dividido en grupos de 12 estudiantes y se realizaron talleres vivenciales centrados en el estudiante, diseñados sobre la base de dinámicas grupales que apunten al aprendizaje experiencial y reflexivo. Esto es facilitado por un tutor, quien promueve la participación activa del estudiante, respetando sus procesos individuales.

La evaluación de los estudiantes se realiza considerando un proceso que contempla evaluación de desempeño, realización de mapa conceptual, autoevaluación, coevaluación y el uso de un diario reflexivo, que corresponde a un registro individual escrito manualmente por el estudiante al finalizar cada sesión de taller. El diario considera: descripción de la sesión (¿de qué se habló?), reflexión (¿qué aprendí?, ¿cómo me sentí?), y transferencia (¿qué aspectos aplico a mi vida futura personal y profesional?).

\section{Método}

La investigación se desarrolla desde un paradigma naturalista con base en el planteamiento de Guba (1981). Utiliza como método cualitativo el estudio de caso único e intrínseco, que busca recoger percepciones de un grupo de personas que vivenciaron una experiencia en común y que permite captar el significado que ellas le dan a este hecho (Vásquez Recio \& Angulo Rasco, 2003).

Se realizó una invitación vía correo electrónico al total de los estudiantes inscritos en la asignatura. La selección de las participantes se hizo con base en aquellos que respondieron a la primera invitación, que estuvieron dispuestos a participar en forma voluntaria, y accedieron a firmar el consentimiento informado y ser parte de una entrevista en profundidad.

Las participantes del estudio fueron seis estudiantes de sexo femenino entre 19 y 24 años, de la carrera de enfermería que cursaron la asignatura durante el segundo semestre del año 2013, número determinado por la saturación de la información en el proceso de recolección de datos.

Se considera como técnica de recolección de datos la entrevista semiestructurada individual al finalizar la asignatura. Para esto se diseña un guion con preguntas orientadoras, que se asocian a los objetivos específicos de la investigación y que fue validado por tres expertos antes del trabajo de campo, considerando la perti- 
nencia con la metodología del estudio, su estructura y narrativa. Se resguardaron los aspectos éticos fundamentales, asegurando la confidencialidad de los datos y garantizando la participación libre y voluntaria en el estudio. Además en el proceso de investigación se lleva un diario del investigador para evidenciar preconcepciones, opiniones y fundamentos en la toma de decisiones.

En el proceso los criterios éticos considerados fueron: negociación, colaboración, confidencialidad, imparcialidad, equidad y compromiso con el conocimiento. Para resguardar la validez y confiabilidad del estudio, se utiliza lo sugerido por Guba (1981): auditoría realizada durante el proceso por la investigadora experta; triangulación de datos, donde se contrastan los datos aportados por los distintos participantes en un proceso comparativo constante; triangulación de investigador, al considerar a otro investigador para contrastar el procesamiento y análisis de los datos; comprobaciones con los participantes, solicitando revisión y validación de las transcripciones a cada participante de manera individual al inicio del proceso de análisis; y análisis de casos negativos, buscando deliberadamente una situación que no se ajuste al patrón del total de entrevistas.

El procesamiento y análisis de datos se realiza con el método comparativo constante (Glaser \& Strauss, 1967), donde se genera una matriz de categorización con enfoque inductivo, con tres núcleos temáticos emergentes, metacategorías asociadas y 241 unidades de significados relacionadas (ver anexo).

\section{Resultados}

Los tres núcleos temáticos que emergen del estudio son desarrollo del proceso reflexi- vo, experiencias de trabajo grupal, y desarrollo de aprendizaje significativo. Estos se describen a continuación, con base en la percepción de los participantes del estudio.

\section{Desarrollo de procesos reflexivos}

Las estudiantes vivencian y desarrollan el proceso reflexivo como una experiencia individual dinámica, recursiva y que para su fluidez requiere un período de maduración, el cual se ve favorecido por diversos aspectos docentes estratégicos que están contenidos en la asignatura, tales como: rol docente, metodología, uso de diario reflexivo y aspectos teóricos trabajados.

El proceso reflexivo es planteado como un proceso a través del cual realizan reflexiones, describiéndolo como un diálogo mental, que se va enriqueciendo al ir integrando las ideas que surgen de las vivencias en cada sesión.

También mencionan etapas dinámicas, sistemáticas, pero no rígidas, a través de las cuales emerge el fenómeno de la reflexión:

1) Surgimiento de ideas que se asocian a vivencias durante la sesión, pero también a recuerdos de la sesión al escribir el diario reflexivo o a vivencias fuera de la sesión.

2) Formulación mental de preguntas a las cuales se someten estas ideas.

3) Contraste de las ideas con pensamientos previos o nuevos que surgen, por ejemplo, al interactuar con otros durante la sesión.

4) Corroboración o modificación de las ideas previas.

5) Surgimiento de nuevas ideas.

6) Estructuración de una opinión o un pensamiento contextualizado con creencias o conceptualizaciones cognitivas propias. 
7) Verbalización de la opinión o idea y/o aplicación consciente de ella.

8) Razonamiento respecto de la verbalización o aplicación de la opinión o idea.

9) Incorporación a las concepciones propias de la opinión o idea, y verbalización o aplicación inconsciente.

Este es un proceso que aumenta en profundidad a medida que avanza la asignatura y que para realizar la reflexión se requiere tranquilidad individual y del entorno, tiempo y disposición para reflexionar.

Respecto a los factores docentes que influyen en los procesos individuales y/o grupales durante la asignatura, existieron temas abordados en las sesiones que fueron más significativos que otros, pues los vincularon a situaciones personales y/o intereses actuales de la formación profesional.

El rol docente se destaca positivamente como facilitador de los procesos individuales y grupales, siendo relevante: manejo de grupo, respeto por los estudiantes, interés por el fomento de la integración, espacios de reflexión y verbalización de opiniones, guía a través de preguntas, actitud de escucha activa, disposición para dar espacio y tiempo necesarios, rol no protagonista, capacidad de realizar intervenciones estratégicas, y flexibilidad.

Además, las estudiantes percibieron de manera positiva una serie de aspectos metodológicos de la asignatura, relacionados con las sesiones de trabajo grupal. Estas son: trabajo en pequeño grupo, tiempo asignado para la sesión, permanecer con el mismo grupo durante el semestre, estructuración de la sesión con capacidad de flexibilidad, dependiendo del proceso grupal, espacio que facilita la reflexión, y sesiones que incorporan dinámicas o actividades grupales de aplica- ción práctica, relacionados con los aspectos teóricos.

Se sugiere que en los aspectos teóricos se contemple el planteamiento de ejemplos o estrategias de aplicación de los temas y que las dinámicas grupales sean contextualizadas a la realidad del estudiante, considerando sus conocimientos y experiencias previas.

El diario reflexivo se percibe como un elemento estratégico de la asignatura, dando continuidad a procesos de reflexión iniciados en las sesiones grupales y permitiendo la expresión de ideas de manera privada. Junto a lo anterior, se identifica una estructura para el desarrollo del diario reflexivo, compuesto por los ítems de descripción, reflexión y transferencia.

\section{Desarrollo de aprendizaje significativo}

Las estudiantes desarrollan aprendizajes significativos, describiéndolos como un proceso dinámico en el cual identifican el aprendizaje inicial, lo aplican en distintos contextos, lo integran como propio y plantean una transferencia de este a nivel personal y profesional futuro. Conceden a la asignatura una connotación positiva, al haber facilitado tales procesos.

Los aprendizajes a los cuales asignan mayor importancia son aquellos que se asocian al desarrollo de capacidades personales, logrando percibir los aprendizajes a través de cambios que evidencian en sus pensamientos y/o conductas a nivel personal y académica, por las experiencias derivadas de la aplicación de ellos.

Se destaca una percepción positiva de los aprendizajes, asumiendo que estos se mantendrán en el tiempo, y asignándole una utilidad integral y amplia, tanto a nivel de la formación profesional, del 
ejercicio profesional futuro y también a nivel personal.

\section{Experiencias en el contexto de trabajo grupal}

Se contempla las experiencias de las estudiantes durante el proceso de conformación del grupo con el cual trabajaron durante el semestre, teniendo en consideración que inicialmente los integrantes tenían diversos niveles de vinculación previa, diferentes expectativas y características personales. Es posible visualizar la generación de procesos individuales que son facilitados por el grupo y que a la vez repercuten en el afiatamiento e interacción grupal, siendo este un proceso progresivo y de evolución individual y colectiva, que incluso se amplía a contextos fuera de la sesión grupal y de la asignatura.

La dinámica grupal se describe como un proceso que va progresando positivamente durante la asignatura y a medida que se van estableciendo vínculos entre los miembros del grupo, durante las sesiones grupales.

Se destacan relatos que tienen relación con la importancia que las estudiantes le atribuyen al grado de conocimiento previo que ellas tienen de miembros del grupo al inicio del curso y las diversas percepciones que manifiestan al respecto.

Dentro de los factores de los estudiantes que influyen en los procesos individuales y/o grupales durante la asignatura, la percepción de confianza al interior del grupo es fundamental para la expresión verbal y emocional durante las sesiones en conjunto. Además, la disposición individual y las características personales son consideradas por las estudiantes como un factor que influye considerablemente en la dinámica grupal.

\section{Discusión y conclusiones}

Mediante esta investigación fue posible la visibilización de áreas de desarrollo del estudiante que desde lo empírico se asumen conocidas a nivel docente y que al explorar en ellas emergen dimensiones no consideradas. Por esto es relevante comprender el desarrollo de los procesos reflexivos y de aprendizaje, como procesos individuales y simultáneos, en que cada uno evoluciona de manera diferente, pero a su vez se influyen mutuamente. De manera paralela, el desarrollo de procesos grupales genera en las estudiantes una serie de reacciones a nivel del comportamiento, interacciones, pensamientos $\mathrm{y}$ emociones, percibiéndose como una amenaza o una oportunidad, dependiendo de las características individuales del estudiante y del nivel de desarrollo de sus procesos reflexivos y de aprendizaje. "La Práctica reflexiva es una modalidad formativa de corte personalista por entender la formación desde la perspectiva de la persona, que parte de su singularidad y por entrelazar profundamente el desarrollo personal con el desarrollo profesional" (Domingo, 2013, p. 386).

El contexto grupal y las metodologías docentes facilitan el desarrollo de los procesos individuales de las estudiantes, quienes en conjunto generan dinámicas grupales que son interactivas entre sí, es decir, el desarrollo de procesos individuales facilita el desarrollo del proceso grupal y este a su vez estimula positivamente el desarrollo individual.

Cabe destacar que la verbalización de opiniones permite visualizar el avance de los tres procesos (reflexivo, trabajo grupal y aprendizaje significativo), pues la construcción de ideas mediante la reflexión, el reconocimiento y apropiación de aprendizajes significativos, permiten al estudian- 
te percibir autoconfianza. Sumado a la percepción de un clima de confianza grupal, facilita la expresión verbal de ideas y opiniones, que a su vez motiva o estimula la expresión verbal en otros miembros del grupo. Además, es posible plantear que en esta dinámica se experimenta reflexión sobre la reflexión sobre la acción (Brockbank \& Mcgill, 2002).

El desarrollo del proceso reflexivo no está directamente relacionado con un aspecto o momento en particular, ni de manera exclusiva con la asignatura o el ámbito académico, sino que es considerado multidimensional y dinámico. Es como un fenómeno que fluye en diferentes contextos y que inicialmente es amplio, ambiguo, incompleto e incluso conflictuante, sin embargo, al ser intencionado de manera tutoriada, va facilitando en el estudiante el desarrollo de habilidades individuales para la reflexión más conscientes y completas, trasferibles a nivel personal, académica y profesional futuro.

La facilitación para el desarrollo de habilidades de reflexión o para desarrollo del proceso reflexivo se asocia a temáticas significativas, pero también al rol de facilitador que cumple el/la docente en el grupo, a los aspectos metodológicos de la asignatura y al uso del diario reflexivo como un elemento estratégico, que apoya y da continuidad al proceso de reflexión del estudiante.

Dentro de los aspectos metodológicos destacados, se encuentra el trabajo en pequeños grupos, la permanencia en el mismo grupo durante el semestre, la estructuración de las sesiones con dinámicas grupales que apoyan la integración de los temas abordados y la flexibilidad del desarrollo de la sesión contextualizada al proceso grupal e individual, dado por el manejo grupal docente.
Las dinámicas grupales son un punto destacado por las estudiantes y desde la fundamentación teórica, se asociaría a que favorecen las cuatro fases del proceso de aprendizaje: activa, reflexiva, teórica y pragmática; y a la vez se consideran los estilos de aprendizajes equivalentes (activo, reflexivo, teórico, pragmático) (González et al., 2016; Lago et al., 2008). Por lo tanto, el diseño de sesiones que incorporan dinámicas grupales que estimulan las cuatro fases y los cuatro estilos de aprendizajes mencionados favorece la participación de manera equitativa de los estudiantes, reconociendo la diversidad, respetando la individualidad $\mathrm{y}$, además, entregando escenarios formativos protegidos para que puedan desarrollar sus áreas de aprendizaje no exploradas.

Otro tema importante son los vínculos de amistad previos con los integrantes del grupo al inicio del proceso. El no tener vinculación anterior es percibido como un factor negativo, que genera actitudes, emociones y pensamientos de diversa magnitud en las estudiantes, lo que depende de las características personales, las expectativas y preconceptos respecto a esta situación. A medida que se va visualizando al otro como conocido y comienzan a establecer vínculos entre ellos, la percepción cambia y este desconocimiento inicial se considera como un aspecto positivo que permite ampliar la red social dentro del curso, estableciendo relaciones de diversa connotación con los integrantes del grupo, llegando incluso a relaciones de amistad posteriores.

La dinámica grupal se presenta como un proceso que progresa en dos niveles. El primero es individual, en el cual las estudiantes experimentan procesos de cambios de actitudes, compromiso, comodidad con el contexto grupal y confianza con los integrantes del grupo. El segundo nivel tiene 
relación con la interacción entre los integrantes del grupo y la influencia positiva mutua, que genera un sentido de pertenencia grupal, constituyéndose en una espiral dialógica, donde la confianza es percibida como un factor fundamental, que favorece los procesos individuales y la dinámica grupal.

El grupo genera un clima, en que existe un compromiso de confidencialidad implícito, que permite la expresión de opiniones y emociones de los integrantes; este clima se vincula con el compromiso de cada integrante, con la dinámica de respeto, de un espacio de libertad de expresión, de aceptación de los procesos individuales propios y de otros. También se relaciona con el permitir y permitirse explorar y desafiarse a compartimientos $\mathrm{u}$ opiniones diferentes en un ambiente percibido como protegido. Lo anterior es coherente con lo planteado por Brockbank \& Mcgill, quienes, citando a Jarvis (1999), mencionan que "...el aprendizaje hay que considerarlo como un fenómeno social y, al mismo tiempo, individual" (Brockbank \& Mcgill, 2002, p. 74).

Las metodologías docentes utilizadas son consideradas estratégicas para el desarrollo de procesos reflexivos, de aprendizajes y grupales; siendo pertinente hacer mención a que los planteamientos teóricos y las dinámicas grupales dirigidas a la integración de estos planteamientos deben estar contextualizados en la realidad de los estudiantes. Deben tomar en consideración sus conocimientos previos, aspectos socioculturales y experiencias académicas, para que los escenarios formativos diseñados realmente faciliten aprendizajes significativos. Además, se sugiere que al plantear aspectos teóricos también se presenten ejemplos o estrategias de aplicación de los temas a lo cotidiano, para que los estudiantes tengan más herramientas al iniciar la reflexión y sus procesos de aprendizaje.

Estos procesos reflexivos son parte del desarrollo de aprendizajes significativos, puesto que la reflexión permite la construcción de pensamientos en forma consciente. Cuando estos interactúan con concepciones previas, movilizan estos conocimientos, son cuestionados o corroborados, fluyendo de esta forma un proceso satisfactorio, dado que las conclusiones o resultados se perciben como un logro propio y desde allí lo significativo de este aprendizaje. Posterior a ello, este aprendizaje pasaría a ser un aprendizaje previo y se aplica de manera fluida e inconsciente a nivel personal y/o académico, siendo posible la proyección al futuro profesional.

Dentro de los aspectos docentes, es interesante que los resultados asociados al uso del diario reflexivo se relacionan de manera muy consistente con la evidencia desde el punto de vista de su forma de utilización, sus beneficios y dificultades (San Rafael Gutiérrez, Siles González, \& Solano Ruiz, 2014). Este registro escrito en un cuaderno se presenta como una herramienta docente que facilita el proceso reflexivo y de aprendizaje significativo, permitiendo materializar los pensamientos en un espacio de intimidad. Se lo considera como un espacio más de reflexión, que permite la sistematización del proceso reflexivo y la visualización de aprendizajes significativos, pudiendo asociarse teóricamente con el concepto de reflexión sobre la acción planteado por Brockbank \& Mcgill (2002) (San Rafael Gutiérrez et al., 2014).

Sin embargo, por la subjetividad que este diario reflexivo tiene, surge el cuestionamiento respecto a su calificación, principalmente del aspecto reflexivo de 
él. Por ello, se hace necesario previo a su incorporación dentro de un programa académico, el análisis de cómo será incluido el diario como herramienta docente. Se debe definir también la orientación que se debe dar a los estudiantes para su uso, sin limitar su creatividad. Paralelamente, se debe reflexionar sobre la coherencia entre el programa, las metodologías docentes, la forma de evaluación, los instrumentos a utilizar y los porcentajes que se aplicarán para la evaluación formativa y/o calificación del diario reflexivo.

En conclusión, este estudio dio la posibilidad de explorar la percepción del desarrollo de los procesos reflexivos de las estudiantes en contraste con aspectos teóricos y a su vez comprender la fluidez, dinamismo e integralidad del fenómeno, pues se evidencia que este no es independiente, más bien interactúa con otros procesos como los de aprendizaje y de desarrollo grupal. Estos hallazgos son relevantes, pues permiten fundamentar la implementación de modificaciones en contextos de innovación curricular, considerando el diseño de programas con enfoque de enseñanza reflexiva, que contemplen metodologías docentes coherentes y que requieren la comprensión por parte del equipo docente de la multidimensionalidad del desarrollo de los procesos reflexivos y su rol influyente como guía o facilitador de ellos.

\section{Referencias}

Asselin, M. E. (2011). Using reflection strategies to link course knowledge to clinical practice: The RN-to-BSN student experience. Journal of Nursing Education, 50(3), 125-133. http://dx.doi.org/10.3928/01484834-20101230-08

Benner, P. (1984). From novice to expert: Excellence and power in clinical nursing practice. Menlo Park, California: Addison-Wesley Publishing Company

Benner, P., Surphen, M., Leonard, V., \& Day, L. (2010). Educating nurses: A call for radical transformation. San Francisco, California: Jossey-Bass.

Brockbank, A. \& Mcgill, I. (2002). Aprendizaje reflexivo en la educación superior. Madrid, España: Morata.

Coll, C., Martin, E., Meuri, T., Miras, M., Onrubia, J., Solé I., \& Zabala, A. (2005). El constructivismo en el aula (15 ${ }^{\mathrm{a}}$ Ed.). Barcelona, España: Graó.

Dahl, H. \& Eriksen, K. A. (2016). Students' and teachers' experiences of participating in the reflection process “THiNK”. Nurse Education Today, 36, 401-406. http://dx.doi.org/10.1016/j.nedt.2015.10.011

Domingo, A. (2013). Práctica reflexiva para docentes: de la reflexión ocasional a la reflexión metodológica. Saarbrücken, Alemania: Publicia.

Glaser, B. G. \& Strauss, A. L. (1967). The discovery of grounded theory: Strategies for qualitative research. New York, New York: Aldine Publishing Company.

González, F., Sáez, K., \& Ramírez, J. (2016). Perfiles de estilos de aprendizaje y rendimiento académico en estudiantes de primer año de enfermería. Ciencia y Enfermería, 22(1), 87-99.

https://dx.doi.org/10.4067/S0717-95532016000100008 
Guba, E. G. (1981).Criteria for assessing the truthworthness of naturalistic inquiries. Educational Communication and Technology Journal, 29(2), 75-91. Recuperado de https://pdfs.semanticscholar.org/8d32/23ed3c76cc4066ec894b5aca51c4f4028b7e.pdf

Jarvis, P. (1999). The Practitioner-Researcher. Developing Theory from Practice. San Francisco, California: Jossey-Bass

Lago, B., Colvin, L., \& Cacheiro, M. (2008). Estilos de aprendizajes y actividades polifásicas: modelo EAAP. Estilos de Aprendizaje, 2(2), 2-21. Recuperado de http://www2.uned.es/revistaestilosdeaprendizaje/numero_2/artigos/1sr2_baldomero.pdf

Medina Moya, J. L. (2002). Práctica educativa y práctica de cuidados enfermeros desde una perspectiva reflexiva. Revista de Enfermería, 15, 21-30. Recuperado de https://is.gd/KA5oZv

Medina Moya, J. L. (2008). La enseñanza en enfermería: de mapas y territorios. Trabajo presentado en XIX Jornadas de Interrelación de Enfermería "El Cuidado de enfermería, un proceso continuado entre niveles", Algüeña, España. Recuperado de http://www.bibliotecadigitalcecova.com/contenido/revistas/cat6/pdf/libro_57.pdf

Medina Moya, J. L., Jarauta Borrasca, B., \& Imbernon Muñoz, F. (2010). La enseñanza reflexiva en la educación superior. Barcelona, España: Octaedro.

Perrenoud, P. (2004). Desarrollar la práctica reflexiva en el oficio de enseñar. Barcelona, España: Graó.

Pina Queirós, P. J. (2015) The knowledge of expert nurses and the practical-reflective rationality. Investigación Educación en Enfermería, 33(1), 83-91. Recuperado de http://www.redalyc.org/pdf/1052/105238622010.pdf

Registered Nurses' Association of Ontario. (RNAO, 2006). Establecimiento de la relación terapéutica: guías de buenas prácticas en enfermería. Ontario, Canadá: Investén. Recuperado de https://is.gd/eU8oCT

San Rafael Gutiérrez, S., Siles González, J., \& Solano-Ruiz C. (2014). El diario del estudiante de enfermería en la práctica clínica frente a los diarios realizados en otras disciplinas. Una revisión integradora. Aquichan, 14(3): 403-416.

http://dx.doi.org/10.5294/aqui.2014.14.3.10

Schön, D. (1992). La formación de profesionales reflexivos: hacia un nuevo diseño de la enseñanza y el aprendizaje en las profesiones. Barcelona, España: Paidós.

Vásquez Recio, R. \& Angulo Rasco, F. (2003). Introducción a los estudios de casos. Los primeros contactos con la investigación etnográfica. Málaga, España: Aljibe. 


\section{Anexo}

Matriz resumen de resultados

\begin{tabular}{|c|c|}
\hline Metacategorías & Unidades de significados destacadas \\
\hline \multicolumn{2}{|l|}{ Desarrollo del proceso reflexivo } \\
\hline \multirow[t]{4}{*}{ Proceso reflexivo } & $\begin{array}{l}\text { (E5-US148) "Comienzo a pensar interna- } \\
\text { mente, hay un diálogo interior". }\end{array}$ \\
\hline & $\begin{array}{l}\text { (E3-US100) "en mi caso me hacía sentido lo } \\
\text { que se iba diciendo, y cosas que a lo mejor } \\
\text { antes tenía una idea contraria como que me } \\
\text { hacían click y decía: eso es, no era lo que yo } \\
\text { pensaba antes, en el caso claro vuelvo a la } \\
\text { asertividad, eso es, no era que estuviera mal } \\
\text { que yo opine distinto, sino que es así porque } \\
\text { somos distintos, como que esas cosas me } \\
\text { iban haciendo click". }\end{array}$ \\
\hline & $\begin{array}{l}\text { (E1-210) "y como que empezaba a juntar } \\
\text { ideas, hasta que... formaba la expresión de lo } \\
\text { que quería decir". }\end{array}$ \\
\hline & $\begin{array}{l}\text { (E3-US83) "yo creo que iba aumentando en } \\
\text { profundidad, o sea, a medida que iban avan- } \\
\text { zando los temas, aunque no fueran más pro- } \\
\text { fundos, como que las reflexiones de uno } \\
\text { mismo lo iban siendo... porque uno se iba } \\
\text { acostumbrando... al menos en mi caso, yo me } \\
\text { iba acostumbrando a ir pensando que me } \\
\text { estaba pasando al reflexionar de estas cosas". }\end{array}$ \\
\hline \multirow[t]{3}{*}{$\begin{array}{l}\text { Factores docentes que influyen } \\
\text { en los procesos individuales y/o } \\
\text { grupales durante la asignatura }\end{array}$} & $\begin{array}{l}\text { (E5-US171) "Creo que los temas que toca- } \\
\text { mos te ayudan para la vida incluso para la } \\
\text { profesión". }\end{array}$ \\
\hline & $\begin{array}{l}\text { (E1-US41) "creo que tienen que ser alguien } \\
\text { capaz de llevar a un grupo y fomentar los } \\
\text { espacios de reflexión y de confianza [do- } \\
\text { cente]". }\end{array}$ \\
\hline & $\begin{array}{l}\text { (E6-US194) "sí yo creo que de partida sea } \\
\text { un grupo chico ideal, el horario también es } \\
\text { ideal porque uno llega con toda la carga de la } \\
\text { semana y un momento donde tú de verdad } \\
\text { bajas las revoluciones te distiendes un poco y } \\
\text { utilizas la parte de una más reflexivas que no }\end{array}$ \\
\hline
\end{tabular}


la utilizas en la semana, uno usa más la parte cognoscitiva, mecánica durante toda la semana y ayuda mucho, las actividades que se hacían súper buenas, me gustaban mucho, porque lo dejaban a uno pensando".

(E2-US68) "Yo creo que las actividades fueron un punto importante, porque, una cosa es el tema, pero lo pueden decir de tal forma y otra forma, pero va a quedar ahí...pero haciendo las actividades como que uno sí o sí la tiene que ir como integrando a algo...y después más encima tener que escribirlo, igual como que se tenía que quedar sí o sí algo de todo lo que hicimos".

(E1-US28) "[diario reflexivo] Lo único que era la lata de escribirlo a mano y todo eso... pero fuera de eso yo encuentro que igual sirve, porque muchas veces uno se queda mucho en el pensamiento y verbalizarlo o escribirlo o contarlo, como que sirve... como que lo hace ser más real.... es un hecho, como que fue, pasó. Entonces en ese sentido yo creo que era útil, porque muchas veces en algunas clases pensábamos y pensábamos, pero muchas veces nadie compartía su experiencia o lo que había reflexionado, entonces como que todo se quedaba en la mente... y de repente escribirlo o recordarlo para poder escribirlo ayudaba a sellar el conocimiento... a dejarlo ahí... ya no se olvida".

Desarrollo de aprendizaje significativo

Aprendizajes relacionados con la

(E1-US40) "yo creo que lo he visto reflejado asignatura en mi capacidad de ahora "de actuar"... como llevar a acción lo que yo quiero, pienso... o verbalizar las cosas. Por ejemplo yo siento que si yo estoy pensando algo que no me parece, poder decirlo... si creo que es una súper buena idea y poder aportar... también... en esas cosas yo creo que lo he visto, en mi relación con las demás personas y en como yo expresarme... cómo me expreso yo con ellos, no ser tan para adentro y no aportar, porque muchas veces no sé si será un tema de 


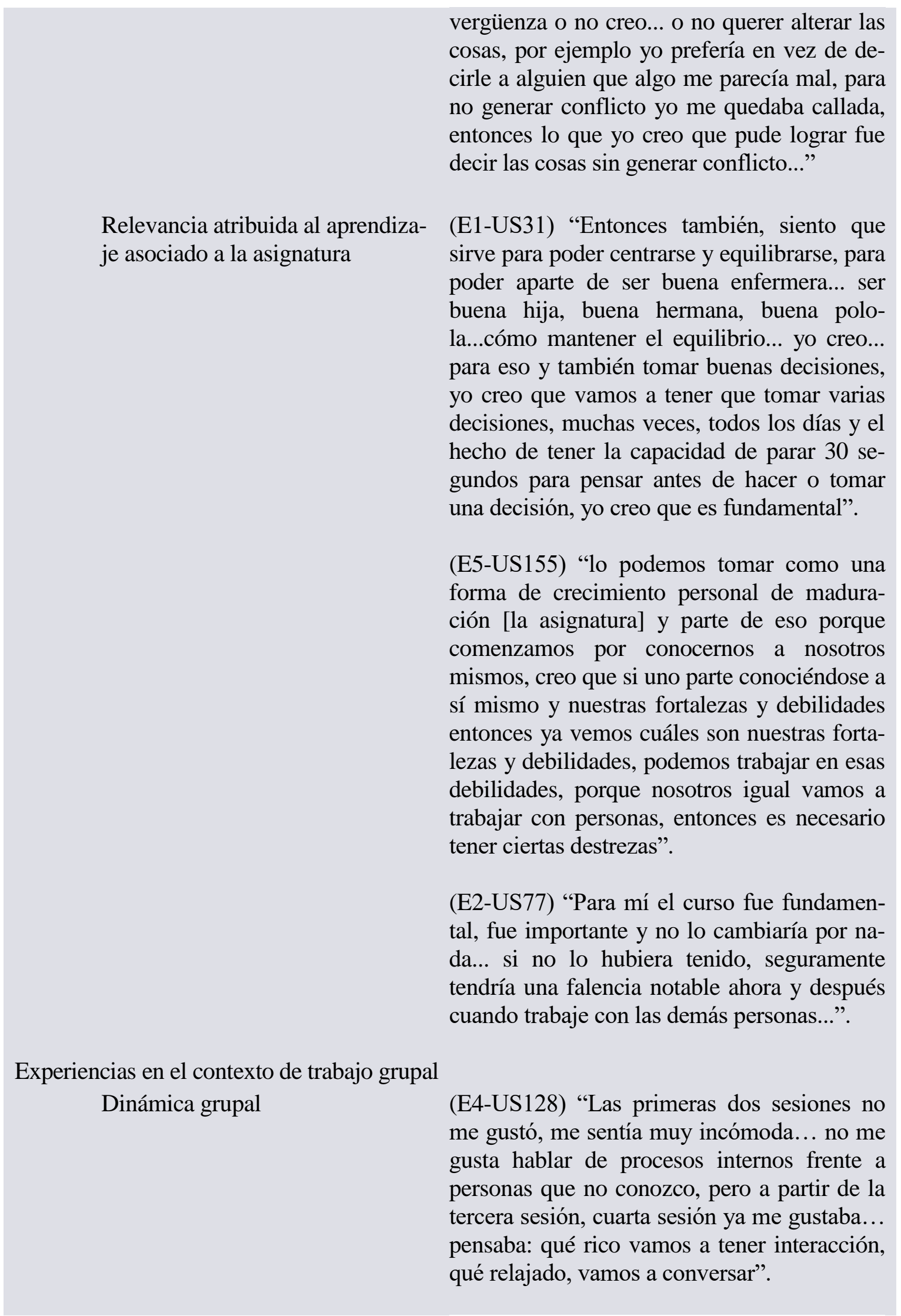


(E6-US191) "yo de hecho con mis compañeros que estuve en el curso de interacción ahora somos amigos".

Factores de los estudiantes que influyen en los procesos individuales y/o grupales durante la asignatura
(E1-US8) "tratamos temas que uno habla con amigos o gente cercana... es difícil... O sea, uno no llega y le cuenta esas cosas a cualquier persona y en ese sentido... a mis compañero y a mí igual me pasó... no sentíamos que eso fuera a salir de ahí... entonces, nos sentíamos con la tranquilidad de poder contarlo... había ese clima, uno empezaba a hablar y como que se olvidaba de que estaba con gente, quizá, que no eran amigos cercanos o familia".

(E1-US32) "Yo creo que lo que entorpecía el trabajo que teníamos o la sesión en general era, por ejemplo que estuviéramos muy cansados... cuando llegábamos el viernes a esa hora y habíamos tenido prueba o algo... se notaba mucho más. No debatíamos tanto, no había tanta actividad, como que queríamos que termináramos luego para ir a descansar... entonces, yo creo que eso de repente interfería porque no aprovechábamos tan bien la instancia como en otras sesiones..." 